\title{
Simple on-line observation and compensation of dead-time effects
}

\author{
S. M. Jung ${ }^{1}$, C. B. Park ${ }^{1}$, J. H. Kim ${ }^{1}$, S. H. Lee ${ }^{1} \&$ H. W. Kim ${ }^{2}$ \\ ${ }^{1}$ Advanced Traction and Noncontact Feeding System Research Team, \\ Korea Railroad Research Institute, Korea \\ ${ }^{2}$ Department of Control and Instrumentation Engineering, \\ Korea National University of Transportation, Korea
}

\begin{abstract}
Generally, there exist voltage differences between the voltage commands and the output voltages of the three-phase pulse-width modulated (PWM) voltage-source inverter (VSI), which are caused by the intended dead-time to prevent the armshort of a leg and the nonlinearities of switching devices. These voltage differences (or voltage distortion) affect the current distortion, torque pulsations, and degradation of control performances. To overcome these drawbacks, a simple and low-cost observation and compensation method of the voltage distortion is proposed. The proposed method is employing model reference adaptive system (MRAS) techniques and does not require any additional hardware circuits such as voltage sensors. The voltage distortions caused by the nonlinearities of the switching devices are estimated by a voltage distortion observer which is based on the electrical model of the PMSM. And the estimated voltage distortions are fed-forward to compensate the voltage commands. In comparison with the previous existing methods, the proposed method has a simple structure and good performances. Also, it can be implemented without any extra devices such as voltage sensors and the computing effort is very small. The execution of the algorithm can be easily embedded in the existing systems without major modification. To show the effectiveness of the proposed method, the experiments are carried out for the digitally controlled PMSM drive system. The experimental results verify the validity of the proposed method and show that the proposed method gives good performance and practical value.

Keywords: dead-time effects, voltage-source inverter, voltage distortion, MRAS.
\end{abstract}




\section{Introduction}

To control traction motor for railway, the pulse width modulated (PWM) voltage source inverter (VSI) is generally used. In general, there exist voltage differences between the voltage commands and the output voltages of the PWM VSI, which are caused by the intended dead-time to prevent the arm-short of a leg and the nonlinearities of switching devices such as voltage drops, turn on/off time, and output voltage transition time. This voltage distortion is severe at low speed range and causes current distortion which may result in unacceptable torque pulsations and degradation of control performance.

To observe and compensate the dead-time effects, various methods have been proposed in the literature [2-7]. However, some require the hardware equipment such as voltage sensors, or some are quite complex to implement and apply the algorithms. To overcome these drawbacks, a simple online observation and compensation method of the dead-time effects is proposed in this paper. The proposed method does not require any additional hardware equipment such as voltage sensors and quite simple to embed the algorithm to an existing system.

\section{Dead-time effects of PWM VSI}

Figure 1 shows the basic configuration of phase A leg in a three-phase PWM VSI. When the system is in the ideal case as can be seen in figure 1(a), the terminal voltage of phase A, $v_{a 0}$, is determined by the phase current $i_{a s}$ and the switching function $\left(S_{a}\right)$ of $Q_{a U}$ and $Q_{a L}$. If the switching function is 1 , which means that $Q_{a U}$ is turned on and $Q_{a L}$ is turned off, the terminal voltage of phase A is equal to $V_{d c} / 2$, where $V_{d c}$ is the DC-link voltage. If the switching function is 0 , which means that $Q_{a U}$ is turned off and $Q_{a L}$ is turned on, $v_{a 0}=-V_{d c} / 2$. The possible terminal voltages according to the switching function and the direction

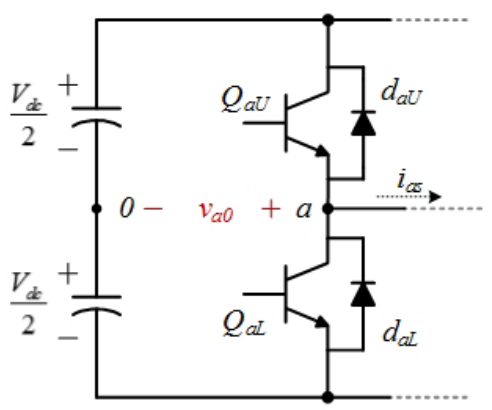

(a)

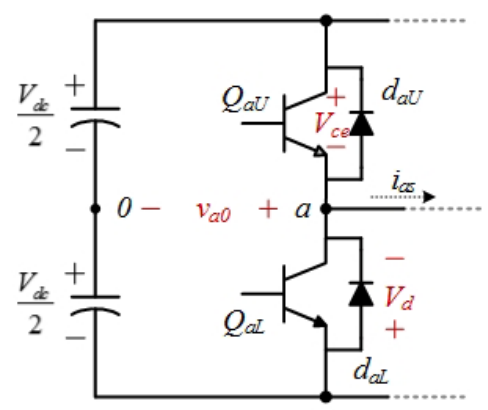

(b)

Figure 1: Basic configuration of phase A leg in a three-phase PWM VSI. (a) Ideal case; (b) Real case including characteristics of the switching devices. 
of phase current in the normal condition are represented in table 1. Unlike the ideal case, however, the real switches in the PWM VSI which have the non-ideal characteristics results in changing the corresponding terminal voltage when the phase current $i_{a s}$ is positive and the switching function $\left(S_{a}\right)$ is 1 . In this case, the terminal voltage of phase $\mathrm{A}$ is not equal to $V_{d c} / 2$, but equal to $V_{d c} / 2-V_{c e}$, where $V_{c e}$ is the forward voltage drop of the body diode in the switch $Q_{a U}$. The equivalent circuit of the real PWM VSI is shown in figure 1(b), and the corresponding terminal voltages are represented in table 2.

Table 1: Terminal voltages of phase $\mathrm{A}$ in ideal case.

\begin{tabular}{c|c|c}
\hline & $S_{a}=1$ & $S_{a}=0$ \\
\hline$i_{a s} \geq 0$ & $v_{a 0}=\frac{V_{d c}}{2}$ & $v_{a 0}=-\frac{V_{d c}}{2}$ \\
\hline$i_{a s}<0$ & $v_{a 0}=\frac{V_{d c}}{2}$ & $v_{a 0}=-\frac{V_{d c}}{2}$ \\
\hline
\end{tabular}

Table 2: Terminal voltages of phase A in real case.

\begin{tabular}{c|c|c}
\hline & $S_{a}=1$ & $S_{a}=0$ \\
\hline$i_{a s} \geq 0$ & $v_{a 0}=\frac{V_{d c}}{2}-V_{c e}$ & $v_{a 0}=-\frac{V_{d c}}{2}-V_{d}$ \\
\hline$i_{a s}<0$ & $v_{a 0}=\frac{V_{d c}}{2}+V_{d}$ & $v_{a 0}=-\frac{V_{d c}}{2}+V_{c e}$ \\
\hline
\end{tabular}

From the above analysis, it is obvious that the phase voltages between the ideal case and real case may have the voltage deviations in the steady state. Based on this fact, the proposed observation and compensation method of deadtime effects indirectly observes these voltage deviations using the analytical model of the PWM VSI.

To take a close look at the effect of the open-circuit fault of a switch on the phase voltages, the knowledge of the relationship between the terminal voltages and the phase voltages is required. This relationship can be represented as follows [1]:

$$
\left[\begin{array}{c}
v_{a 0} \\
v_{b 0} \\
v_{c 0}
\end{array}\right]=\left[\begin{array}{c}
v_{a s} \\
v_{b s} \\
v_{c s}
\end{array}\right]+v_{s 0}
$$




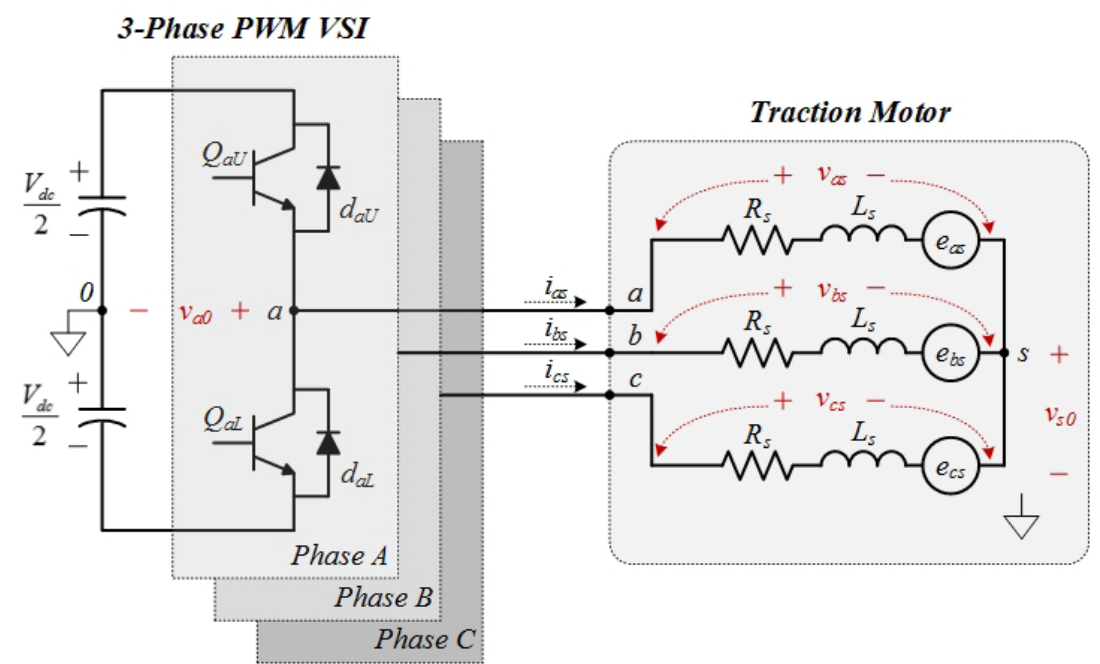

Figure 2: Relationship between terminal voltages and phase voltages.

where $v_{a s}, v_{b s}$, and $v_{c s}$ are the phase voltages and $v_{s 0}$ is the neutral to centre voltage (as shown in figure 2). In a three-phase three-wire system, the following condition by Kirchoff's law is satisfied as

$$
i_{a s}+i_{b s}+i_{c s}=0
$$

Also, the sum of each phase back-EMF is equal to zero at any instant under the assumption that the air-gap magnetic flux distribution is a sinusoid. From eqn. (2) and this assumption, the following condition is satisfied for the PMSM as

$$
v_{a s}+v_{b s}+v_{c s}=R_{s}\left(i_{a s}+i_{b s}+i_{c s}\right)+L_{s} \frac{d}{d t}\left(i_{a s}+i_{b s}+i_{c s}\right)+\left(e_{a s}+e_{b s}+e_{c s}\right)=0
$$

where $R_{s}$ is a stator resistance, $L_{s}$ is a stator inductance, and $e_{a s}, e_{b s}$, and $e_{c s}$ represent the corresponding phase back-EMFs, respectively. From eqn. (1) to eqn. (3), the neutral to centre voltage, $v_{s 0}$, becomes

$$
v_{s 0}=\frac{1}{3}\left(v_{a 0}+v_{b 0}+v_{c 0}\right) .
$$

Therefore, the relationship between the terminal voltages and the phase voltages can be represented as follows: 


$$
\left[\begin{array}{l}
v_{a s} \\
v_{b s} \\
v_{c s}
\end{array}\right]=\frac{1}{3}\left[\begin{array}{ccc}
2 & -1 & -1 \\
-1 & 2 & -1 \\
-1 & -1 & 2
\end{array}\right]\left[\begin{array}{l}
v_{a 0} \\
v_{b 0} \\
v_{c 0}
\end{array}\right] .
$$

On the other hand, the dead-time effects can be represented by a deviation value from the terminal voltages of the PWM VSI. If $\Delta v_{a 0}, \Delta v_{a 0}$, and $\Delta v_{a 0}$ are considered as representing the voltage deviations due to the dead-time effects, the phase voltages are represented as follows:

$$
\left[\begin{array}{c}
v_{a s_{-} \text {real }} \\
v_{b_{\text {_real }}} \\
v_{c s_{\text {rreal }}}
\end{array}\right]=\frac{1}{3}\left[\begin{array}{ccc}
2 & -1 & -1 \\
-1 & 2 & -1 \\
-1 & -1 & 2
\end{array}\right]\left[\begin{array}{c}
v_{a 0} \pm \Delta v_{a 0} \\
v_{b 0} \pm \Delta v_{b 0} \\
v_{c 0} \pm \Delta v_{c 0}
\end{array}\right]
$$

where $v_{a s_{-} \text {real }}, v_{b s_{-} \text {real }}$, and $v_{c s_{-} \text {real }}$ are the phase voltages in the real case. After some calculations, eqn. (6) can be expressed as

$$
\begin{aligned}
{\left[\begin{array}{c}
v_{a s_{-} \text {real }} \\
v_{b s_{-} \text {real }} \\
v_{c s_{-} \text {real }}
\end{array}\right] } & =\left[\begin{array}{c}
v_{a s} \\
v_{b s} \\
v_{c s}
\end{array}\right]+\frac{1}{3}\left[\begin{array}{ccc}
2 & -1 & -1 \\
-1 & 2 & -1 \\
-1 & -1 & 2
\end{array}\right]\left[\begin{array}{l} 
\pm \Delta v_{a 0} \\
\pm \Delta v_{a 0} \\
\pm \Delta v_{a 0}
\end{array}\right] \\
& =\left[\begin{array}{c}
v_{a s} \\
v_{b s} \\
v_{c s}
\end{array}\right]+\left[\begin{array}{c}
\Delta v_{a s_{-} \text {dist }} \\
\Delta v_{b s_{-} \text {dist }} \\
\Delta v_{c s_{-} \text {dist }}
\end{array}\right]
\end{aligned}
$$

where $\Delta v_{\text {as_dist }}, \Delta v_{b s \_d i s t}$, and $\Delta v_{c s_{\text {_dist }}}$ are the phase voltage deviations introduced by the dead-time effects. As can be seen in eqn. (7), the phase voltage in the real case can be divided into two parts. The first term of eqn. (7), $v_{k s}(k=\{a, b, c\})$, are the ideal phase voltages and the second term of eqn. (7), $\Delta v_{k s}$ dist $(k=\{a, b$, $c\})$, are the voltage deviations due to the dead-time effects. These phase voltage deviations can be considered as 'voltage distortions' induced by the dead-time effects and observed from the machine parameters, which is discussed in the following.

The voltage distortions in the $a b c$ frame can be transformed to the rotor reference frame by using the relationship [1] as 


$$
\begin{gathered}
{\left[\begin{array}{c}
v_{q_{-} \text {real }} \\
v_{d_{-} \text {real }}
\end{array}\right]=\mathrm{T}\left(\theta_{e}\right)\left[\begin{array}{c}
v_{\text {as_real }} \\
v_{b_{s_{-} \text {real }}} \\
v_{\text {cs_real }}
\end{array}\right],} \\
\mathrm{T}\left(\theta_{e}\right)=\frac{2}{3}\left[\begin{array}{ccc}
\cos \theta_{e} & \cos \left(\theta_{e}-\frac{2 \pi}{3}\right) & \cos \left(\theta_{e}+\frac{2 \pi}{3}\right) \\
\sin \theta_{e} & \sin \left(\theta_{e}-\frac{2 \pi}{3}\right) & \sin \left(\theta_{e}+\frac{2 \pi}{3}\right)
\end{array}\right]
\end{gathered}
$$

where $\theta_{e}$ is the electrical angular position of the rotor. Using from eqn. (7) to eqn. (9), the voltage distortions in the rotor reference frame can be represented as

$$
\left[\begin{array}{c}
v_{q_{-} \text {real }} \\
v_{d_{-} \text {real }}
\end{array}\right]=\mathrm{T}\left(\theta_{e}\right)\left[\begin{array}{c}
v_{a s}+\Delta v_{a s_{-} \text {dist }} \\
v_{b s}+\Delta v_{b s_{-} \text {dist }} \\
v_{c s}+\Delta v_{c s_{-} \text {dist }}
\end{array}\right]=\left[\begin{array}{c}
v_{q} \\
v_{d}
\end{array}\right]+\left[\begin{array}{c}
\Delta v_{q_{-} \text {dist }} \\
\Delta v_{d_{-} \text {dist }}
\end{array}\right]
$$

where $v_{q_{-} \text {real }}$ and $v_{d_{-} \text {real }}$ are the $q$ - and $d$-axis voltages in the real case, $v_{q}$ and $v_{d}$ are the ideal $q$ - and $d$-axis voltages, and $\Delta v_{q_{-} \text {dist }}$ and $\Delta v_{d_{-} \text {dist }}$ are the $q$ - and $d$-axis voltage deviations or distortions due to the dead-time effects. The stator voltages in the rotor reference frame after the fault occurrence can also be represented as two parts. One is the ideal $q$ - and $d$-axis voltages and the other is the voltage deviations caused by the dead-time effects.

\section{Proposed on-line observation and compensation of dead-time effects}

The current dynamics of a PMSM including the voltage distortions caused by the dead-time effects can be represented as follows [1]:

$$
\left[\begin{array}{c}
\frac{d i_{q_{-} \text {real }}}{d t} \\
\frac{d i_{d_{-} \text {real }}}{d t}
\end{array}\right]=\left[\begin{array}{cc}
-\frac{R_{s}}{L_{s}} & -\omega_{e} \\
\omega_{e} & -\frac{R_{s}}{L_{s}}
\end{array}\right]\left[\begin{array}{c}
i_{q_{-} \text {real }} \\
i_{d_{-} \text {real }}
\end{array}\right]+\frac{1}{L_{s}}\left[\begin{array}{c}
v_{q}+\Delta v_{q_{-} \text {dist }} \\
v_{d}+\Delta v_{d_{-} \text {dist }}
\end{array}\right]+\left[\begin{array}{c}
\lambda_{m} \omega_{e} \\
L_{s} \\
0
\end{array}\right]
$$

where $i_{q_{-} \text {real }}$ and $i_{d_{-} \text {real }}$ are the $q$ - and $d$-axis currents, $\omega_{e}$ is the electrical rotor angular speed, $\lambda_{m}$ is the flux linkage established by the permanent magnet, respectively. As can be seen in eqn. (11), the motor currents are affected by the voltage distortions caused by the dead-time effects. 


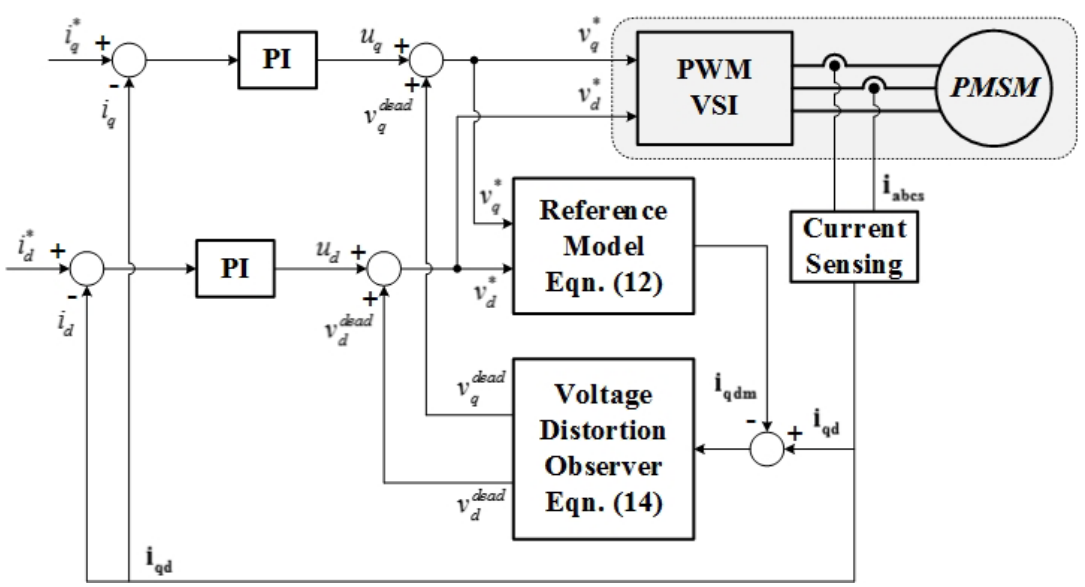

Figure 3: Current control block diagram using the proposed voltage distortion observer for the compensation of the dead-time effects.

For the reference model of the MRAS, it is assumed that the voltage distortions due to the dead-time effects are zero and the system is in the ideal case. In this condition, the calculated current dynamics using the nominal parameters can also be represented as follows:

$$
\left[\begin{array}{c}
\frac{d i_{q m}}{d t} \\
\frac{d i_{d m}}{d t}
\end{array}\right]=\left[\begin{array}{cc}
-\frac{R_{s 0}}{L_{s 0}} & -\omega_{e} \\
\omega_{e} & -\frac{R_{s 0}}{L_{s 0}}
\end{array}\right]\left[\begin{array}{l}
i_{q} \\
i_{d}
\end{array}\right]+\frac{1}{L_{s 0}}\left[\begin{array}{c}
v_{q}^{*} \\
v_{d}^{*}
\end{array}\right]+\left[\begin{array}{c}
-\frac{\lambda_{m 0} \omega_{e}}{L_{s 0}} \\
0
\end{array}\right]
$$

where $v_{q}{ }^{*}$ and $v_{d}{ }^{*}$ are the $q$ - and $d$-axis stator voltage commands, $i_{q m}$ and $i_{d m}$ are the $q$ - and $d$-axis currents of the model, respectively, and the subscript " 0 " represents the nominal value.

From eqn. (11) and eqn. (12), the voltage distortions in the rotor reference frame caused by the dead-time effects can be obtained as follows:

$$
\begin{aligned}
& v_{q}^{\text {dead }}=v_{q}^{*}-v_{q_{-} \text {real }}=-L_{s}\left(\frac{d i_{q_{-} \text {real }}}{d t}-\frac{d i_{q m}}{d t}\right) \\
& v_{d}^{\text {dead }}=v_{d}^{*}-v_{d_{-} \text {real }}=-L_{s}\left(\frac{d i_{d_{-} \text {real }}}{d t}-\frac{d i_{d m}}{d t}\right)
\end{aligned}
$$

where it is assumed that the nominal parameters $R_{s 0}, L_{s 0}$, and $\lambda_{m 0}$ are identical to the real values $R_{s}, L_{s}$, and $\lambda_{m}$, respectively. The average voltage distortions over the $k^{\text {th }}$ PWM step can be derived from eqn. (13) as follows: 
Table 3: Specifications of the test PMSM.

\begin{tabular}{|c|c|c|c|}
\hline Rated Power & $750[\mathrm{~W}]$ & \# of poles & 8 \\
\hline Rated Torque & $2.4[\mathrm{Nm}]$ & Rated Speed & $3000[\mathrm{r} / \mathrm{min}]$ \\
\hline $\begin{array}{c}\text { Phase } \\
\text { Resistance }\end{array}$ & $0.49[\Omega]$ & Rated Current & $6.0[\mathrm{~A}]$ \\
\hline Linkage Flux & $0.0667[\mathrm{~Wb}]$ & $\begin{array}{l}\text { Phase } \\
\text { Inductance }\end{array}$ & $6.9[\mathrm{mH}]$ \\
\hline
\end{tabular}

Table 4: Specifications of the three-phase PWM VSI.

\begin{tabular}{cc|cc}
\hline $\begin{array}{c}\text { DC-link } \\
\text { Voltage }\end{array}$ & $300[\mathrm{~V}]$ & $\begin{array}{c}\text { Switching } \\
\text { Frequency }\end{array}$ & $11[\mathrm{kHz}]$ \\
\hline $\begin{array}{c}\text { Dead-time } \\
\text { Turn-on Time }\end{array}$ & $2.8[\mathrm{usec}]$ & $\begin{array}{c}\text { Switching } \\
\text { Device }\end{array}$ & IGBT \\
$\begin{array}{c}\text { Saturation } \\
\text { Voltage }^{\dagger}\end{array}$ & $25[\mathrm{nsec}]$ & $\begin{array}{c}\text { Turn-off } \\
\text { Time }^{\dagger}\end{array}$ & $115[\mathrm{nsec}]$ \\
\hline
\end{tabular}

${ }^{\dagger}$ Fairchild datasheet (FGH40N60SFD).

$$
\begin{aligned}
& v_{q}^{\text {dead }}(k)=v_{q}^{*}(k)-v_{q_{-} \text {real }}(k)=-L_{s}\left(\frac{i_{q_{\_} \text {real }}(k)-i_{q m}(k)}{T_{s}}\right) \\
& v_{d}^{\text {dead }}(k)=v_{d}^{*}(k)-v_{d_{-} \text {real }}(k)=-L_{s}\left(\frac{i_{d_{\_} \text {real }}(k)-i_{d m}(k)}{T_{s}}\right)
\end{aligned} .
$$

The model currents $i_{q m}(k)$ and $i_{d m}(k)$ in eqn. (14) can be obtained from the discrete form of eqn. (12).

By the feed-forward compensation manner, the observed dead-time effect in eqn. (14) is added to the voltage commands. The voltage commands with the compensation of the dead-time effect are represented as follows: 


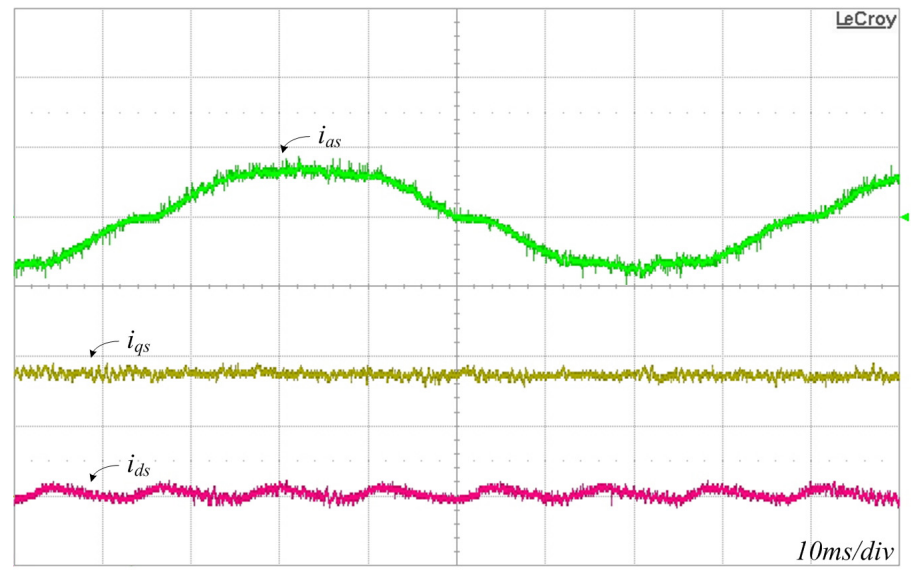

(a)

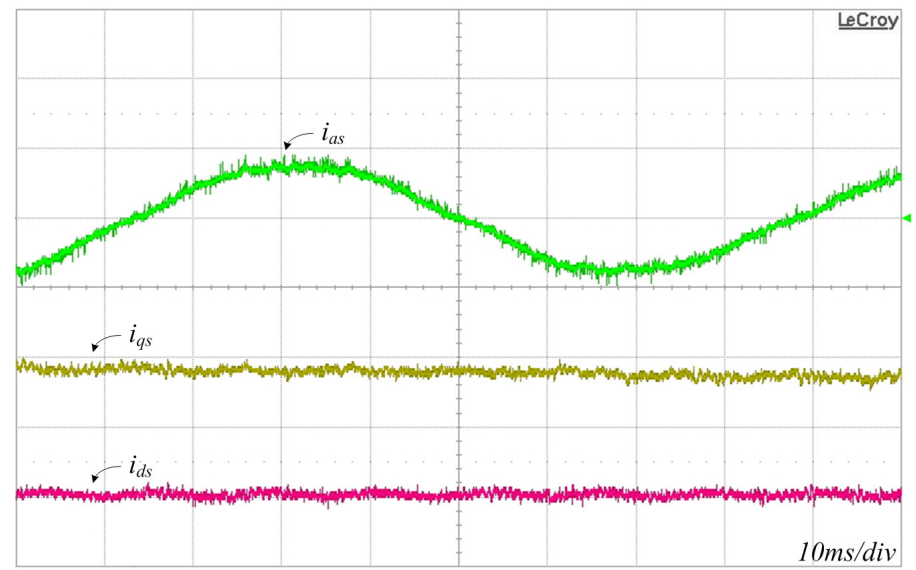

(b)

Figure 4: Experimental results of the current waveforms. (a) Without dead-time compensation. (b) With the proposed voltage distortion observer.

$$
\begin{aligned}
& v_{q}^{*}(k)=u_{q}(k)+e_{q}(k)+v_{q}^{\text {dead }}(k) \\
& v_{d}^{*}(k)=u_{d}(k)+e_{d}(k)+v_{d}^{\text {dead }}(k)
\end{aligned}
$$

where $u_{q}$ and $u_{d}$ are the results of decoupled PI current control in $q$ - and $d$-axis, respectively, $e_{q}=L_{s} \omega_{e} i_{d}+\lambda_{m} \omega_{e}, e_{d}=-L_{s} \omega_{e} i_{q}$, and $v_{q}^{\text {dead }}$ and $v_{d}^{\text {dead }}$ are the compensation voltages in $q$ - and $d$-axis from eqn. (14), respectively. The overall block diagram of the compensation for the dead-time effect is shown in figure 3 . 
In order to confirm the feasibility of the fault diagnosis algorithm, the experiments have been realized scaled-down model. The parameters related to the test motor and three-phase PWM converter are represented in table 3 and table 4, respectively. The converter is composed of six IGBTs, FGH40N60SFD from Fairchild, Corp. The switching frequency of the converter is $11 \mathrm{kHz}$. All the control laws proposed in this dissertation have been realized by using a single microcontroller unit TMS320F28335 from Texas Instruments, Inc. The sampling rate of phase currents is identical to the switching frequency.

The experimental result for the compensation of the dead-time effect is shown in figure 4. The phase A current waveform without the dead-time compensation is distorted, but this is almost sinusoids with the proposed compensation method. Therefore, the proposed method can be used for the dead-time.

\section{Conclusion}

In this paper, a simple online observation and compensation method of the deadtime effects is proposed, which will improve the current distortion of the PWM VSI. As a first step, the dead-time effects is analysed, which changes the corresponding terminal voltage and introduces the voltage deviations or voltage distortions to each phase voltage. By observing these voltage distortions achieved by the simple MRAS-based voltage distortion observer, the current distortion can be significantly reduced. The proposed compensation method is also quite simple so that the algorithm can be easily embedded in the existing systems without major modifications. To show the effectiveness of the proposed method, the experiments are carried out for the digitally controlled drive system. The results verify the validity of the proposed method and show that the proposed method gives the good performance and practical value.

\section{Acknowledgements}

This work was supported by the Human Resources Development program (No. 20134030200310) of the Korea Institute of Energy Technology Evaluation and Planning (KETEP) grant funded by the Korea government Ministry of Trade, Industry and Energy.

\section{References}

[1] P.C. Krause, O. Wasynczuk, and S. D. Sudhoff, Analysis of Electric Machinery, IEEE Power Eng. Soc., 1995.

[2] J. Holtz, J. Quan (2002) Sensorless vector control of induction motors at very low speed using a nonlinear inverter model and parameter identification, IEEE Transactions on Industrial Applications, 38(2), pp. 1087-1095. 
[3] J. W. Choi, S. K. Sul (1996) Inverter output voltage synthesis using novel dead time compensation, IEEE Transactions on Power Electronics, 11(2), pp. 221-227.

[4] A. R. Munoz, T. A. Lipo (1999) On-line dead-time compensation technique for open-loop PWM-VSI drives, IEEE Transactions on Power Electronics, 14(4), pp. 683-689.

[5] F. Blaabjerg, J. K. Pederson, P. Thoegersen (1997) Improved modulation techniques for PWM-VSI drives, IEEE Transactions on Industrial Electronics, 44(1), pp. 87-95.

[6] H. S. Kim, K. H. Kim, M. J. Youn (2003) On-line dead-time compensation method based on time delay control, IEEE Transactions on Control System Technology, 11(2), pp. 279-285.

[7] H. W. Kim, H. S. Kim, M. J. Youn (2004) Online observation and compensation of voltage distortion in PWM VSI for PMSM, IEE Electric Power Applications, 151(5), pp. 534-542. 